\title{
Empty Simplices of Polytopes and Graded Betti Numbers
}

\author{
Uwe Nagel
}

Received: 12 September 2005 / Revised: 13 February 2006

(C) Springer Science+Business Media, LLC 2008

\begin{abstract}
The conjecture of Kalai, Kleinschmidt, and Lee on the number of empty simplices of a simplicial polytope is established by relating it to the first graded Betti numbers of the polytope and applying a result of Migliore and the author. This approach allows us to derive explicit optimal bounds on the number of empty simplices of any given dimension. As a key result, we prove optimal bounds for the graded Betti numbers of any standard graded $K$-algebra in terms of its Hilbert function.
\end{abstract}

\section{Introduction}

Let $P \subset \mathbb{R}^{d}$ be a simplicial $d$-polytope, i.e., the $d$-dimensional convex hull of finitely many points in $\mathbb{R}^{d}$ such that all its faces are simplices. The simplest combinatorial invariant of $P$ is its $f$-vector $f=\left(f_{-1}, f_{0}, \ldots, f_{d-1}\right)$ where $f_{-1}:=1$ and $f_{i}$ is the number of $i$-dimensional faces of $P$ if $i \geq 0$. In [14] McMullen conjectured a characterization of the possible $f$-vectors. In order to state his conjecture we use an equivalent set of invariants, the $h$-vector $\underline{h}:=\left(h_{0}, \ldots, h_{s}\right)$. It is defined as the sequence of coefficients of the polynomial

$$
\sum_{j=0}^{s} h_{j} z^{j}:=\sum_{j=0}^{d} f_{j-1} \cdot z^{j}(1-z)^{d-j}
$$

The $f$-vector can be recovered from the $h$-vector because

\footnotetext{
U. Nagel (两)

Department of Mathematics, University of Kentucky, 715 Patterson Office Tower, Lexington,

KY 40506-0027, USA

e-mail: uwenagel@ms.uky.edu
} 


$$
f_{j-1}=\sum_{i=0}^{j}\left(\begin{array}{l}
d-i \\
j-i
\end{array}\right) h_{i} .
$$

Using $h$-vectors we can state McMullen's conjecture which has become a proven statement by combining the results of Billera and Lee [2] and Stanley [20] (see also [15]).

Theorem 1.1 (g-Theorem) A sequence $\underline{h}=\left(h_{0}, \ldots, h_{s}\right)$ of positive integers is the $h$-vector of a simplicial d-polytope if and only if $s=d$ and $\underline{h}$ is an SI-sequence, i.e., h satisfies:

(i) (Dehn-Sommerville equations) $h_{i}=h_{d-i}$ for $i=0, \ldots, d$.

(ii) $\underline{g}:=\left(h_{0}, h_{1}-h_{0}, \ldots, h_{\lfloor d / 2\rfloor}-h_{\lfloor d / 2\rfloor-1}\right)$ is an $O$-sequence.

Being an O-sequence is a purely numerical condition (see Sect. 3). Note that O-sequences are precisely the Hilbert functions of Artinian standard graded $K$-algebras.

In order to prove sufficiency of these conditions, in [2] Billera and Lee construct, for each SI-sequence $\underline{h}:=\left(h_{0}, \ldots, h_{d}\right)$, a certain simplicial $d$-polytope $P_{\mathrm{BL}}(\underline{h})$ whose $h$-vector is the given SI-sequence $\underline{h}$. The Billera-Lee polytopes are rather particular which has led to expectations that they have some extremal properties. In order to state one such instance recall (see [11]) that an empty simplex of the polytope $P$ is a smallest subset $S$ of the vertex set of $P$ such that $S$ is not a face of $P$, but each proper subset of $S$ is a face of $P$. Sometimes, empty simplices are called missing faces. They are just minimal non-faces of the vertex set of $P$. Empty simplices play an important role in the classification of polytopes (see, e.g., [9] and Remark 4.19). In [10] Kalai states as Conjecture 2:

Conjecture 1.2 (Kalai, Kleinschmidt, Lee) For all simplicial d-polytopes with prescribed $h$-vector $\underline{h}$, the number of $j$-dimensional empty simplices is maximized by the Billera-Lee polytope $P_{\mathrm{BL}}(\underline{h})$.

Kalai has pointed out in Theorem 19.5.35 of [11] that this conjecture is a consequence of results by Migliore and the author [16], but his argument needs some adjustment. The starting point of this note is to point out in detail the connection to the results in [16] that leads to a proof of the conjecture in Theorem 2.3.

The construction of the Billera-Lee polytopes is rather involved. In general, the number of empty $j$-simplices of a given Billera-Lee polytope $P_{\mathrm{BL}}(\underline{h})$ has not been known. Hence, the proof of Conjecture 1.2 leaves open the problem of giving an explicit bound in terms of the $h$-vector. The bulk of this paper is devoted to solving this problem. The key is given by our proof of Conjecture 1.2. It identifies the number of missing $j$-simplices of the polytope $P$ with a certain graded Betti number of its Stanley-Reisner ring $K[P]$. Since the $h$-vector of $P$ is determined by the Hilbert function of $K[P]$, we are led to consider the problem of finding sharp upper bounds for the graded Betti numbers of the Stanley-Reisner ring $K[P]$ in terms of its Hilbert 
function. We solve this problem in Sect. 3 in greater generality, namely for Gorenstein algebras with the Weak Lefschetz property (Theorem 3.17). Its proof requires explicit bounds for all graded Betti numbers of any standard graded $K$-algebra $A$ in terms of its Hilbert function. These are established in Theorem 3.12. They are optimal. Because of the importance of graded Betti numbers, it seems fair to expect that Theorem 3.12 will find applications in other contexts as well.

In Sect. 4 we apply the results of Sect. 3 to derive explicit optimal bounds for the number of missing $j$-simplices of a simplicial polytope in terms of its $g$-vector (see Corollary 4.6). Note that the $g$-vector is easily obtained from the $h$-vector (Definition 4.2). We conclude with some applications. Let $N(k)$ be the number of empty $j$-simplices of a simplicial polytope $P$ such that $j \leq k$. In Theorem 4.15 we establish an upper bound for $N(k)$ that depends on $k$, the dimension $d$, and the number of vertices $f_{0}$ of the polytope. For each given triple $\left(k, d, f_{0}\right)$, this bound is attained when $P$ is a certain Billera-Lee polytope. For example, the bound for the number of empty edges reads as

$$
N(1) \leq \begin{cases}f_{0}\left(f_{0}-3\right) / 2 & \text { if } d=2 \\
\left(\begin{array}{c}
f_{0}-d \\
2
\end{array}\right) & \text { if } d \geq 3\end{cases}
$$

It is always sharp if the polytope is stacked (see Example 4.18(ii)). McMullen's famous Upper Bound Theorem [13] states that the cyclic polytope $C\left(f_{0}, d\right)$ has the maximal $f$-vector among all simplicial $d$-polytopes with $f_{0}$ vertices. Our Theorem 4.15 shows that it also has the maximal total number of empty simplices among these polytopes (Example 4.18(i)).

As a consequence of Theorem 4.15 , for a simplicial $d$-polytope we obtain a bound for the number $N(k)$ that depends only on $k$ and $f_{0}-d$ (Corollary 4.16). Following Kalai [10], such a bound is the key to a central result of Perles [18] in the theory of arbitrary polytopes with "few vertices" (see Remark 4.19). Finally, we show that very little information on the $g$-vector is sufficient to bound the number of empty $j$-simplices of a simplicial $d$-polytope if $d$ is large enough (Corollary 4.22). This result slightly corrects and improves Theorem 3.8 of [10].

\section{The Conjecture of Kalai, Kleinschmidt, and Lee}

The goal of this section is to prove Conjecture 1.2. To this end we need some more notation. Let $P$ be a simplicial $d$-polytope. Denote its vertex set by $\left\{v_{1}, \ldots, v_{f_{0}}\right\}$ and let $R:=K\left[x_{1}, \ldots, x_{f_{0}}\right]$ be the polynomial ring in $f_{0}$ variables over an arbitrary field $K$. Then the Stanley-Reisner ring of $P$ is $K[P]:=R / I_{P}$ where the Stanley-Reisner ideal is generated by all square-free monomials $x_{i_{1}} x_{i_{2}} \cdots x_{i_{t}}$ such that $\left\{v_{i_{1}}, v_{i_{2}}, \ldots, v_{i_{t}}\right\}$ is not a face of $P$. It is well-known (see Corollary 5.6.5 of [3]) that $K[P]$ is a Gorenstein $\operatorname{ring}$ of dimension $d=\operatorname{dim} P$. Since $h_{1}=f_{0}-d$, its minimal graded free resolution is of the form

$$
0 \rightarrow \bigoplus_{j \in \mathbb{Z}} R(-j)^{\beta_{h_{1}, j}^{K}(P)} \rightarrow \cdots \rightarrow \bigoplus_{j \in \mathbb{Z}} R(-j)^{\beta_{1, j}^{K}(P)} \rightarrow R \rightarrow R / I_{P} \rightarrow 0 .
$$

The non-negative integers $\beta_{i, j}^{K}(P)=\operatorname{dim}_{K}\left[\operatorname{Tor}_{i}^{R}(K[P], K)\right]_{j}, i, j \in \mathbb{Z}$, are called the graded Betti numbers of $P$. 
The following result is shown in [16]:

Theorem 2.1 Let $K$ be a field of characteristic zero and let $P$ be a simplicial $d$ polytope with $h$-vector $\underline{h}$. Then we have for all integers $i, j$,

$$
\beta_{i, j}^{K}(P) \leq \beta_{i, j}^{K}\left(P_{\mathrm{BL}}(\underline{h})\right)
$$

Proof The claim is a consequence of Theorem 9.6 of [16], because its proof shows (see page 57) that the extremal polytope that is not specified in part (b) of this theorem is indeed the Billera-Lee polytope $P_{\mathrm{BL}}(\underline{h})$.

Remark 2.2 The assumption on the characteristic of the field $K$ is needed to ensure that the Stanley-Reisner ring $K[P]$ has the so-called Weak Lefschetz property (see Sect. 3). This property also plays a crucial role in Stanley's necessity part of the g-Theorem in [20].

The conjecture of Kalai, Kleinschmidt, and Lee now follows easily.

Theorem 2.3 For all simplicial polytopes with prescribed $h$-vector $\underline{h}$, the number of $j$-dimensional empty simplices is maximized by the Billera-Lee polytope $P_{\mathrm{BL}}(\underline{h})$.

Proof It follows from its definition that $\beta_{1, j}^{K}(P)$ is the number of minimal generators of degree $j$ of the Stanley-Reisner ideal $I_{P}$. A $j$-dimensional empty simplex $S$ of $P$ corresponds to a monomial $m_{S}$ of degree $j+1$ in $I_{P}$. Since each proper subset of $S$ is a face of $P$, the monomial $m_{S}$ is not a proper multiple of any monomial in $I_{P}$, i.e., $m_{S}$ is a minimal generator of $I_{P}$. Therefore, the conjecture of Kalai, Kleinschmidt, and Lee is a consequence of Theorem 2.1 applied with $i=1$.

The combinatorial interpretation of the first Betti numbers allows us to drop the assumption on the characteristic in Theorem 2.1 for certain Betti numbers.

Corollary 2.4 Let $P$ be a simplicial d-polytope with $h$-vector $\underline{h}$. Then we have for all integers $j$,

$$
\beta_{1, j}^{K}(P) \leq \beta_{1, j}^{K}\left(P_{\mathrm{BL}}(\underline{h})\right), \quad \beta_{h_{1}-1, j}^{K}(P) \leq \beta_{h_{1}-1, j}^{K}\left(P_{\mathrm{BL}}(\underline{h})\right),
$$

and

$$
\beta_{h_{1}, j}^{K}(P)= \begin{cases}0 & \text { if } j \neq h_{1}+d \\ 1 & \text { if } j=h_{1}+d\end{cases}
$$

Proof Denote by $n_{j}(P)$ the number of empty $j$-simplices of $P$. We have seen that, for every field $K$,

$$
n_{j-1}(P)=\beta_{1, j}^{K}(P) .
$$

Let $K$ be a field of characteristic zero. Then Theorem 2.3 provides

$$
n_{j-1}(P) \leq n_{j-1}\left(P_{\mathrm{BL}}(\underline{h})\right) \text {. }
$$


Let now $K$ be an arbitrary field. Then, applying the above equality again, the claim for the first Betti numbers follows.

Since $K[P]$ is a Gorenstein ring, its minimal free resolution is self-dual. In particular, for all integers $i, j$, we have

$$
\beta_{i, j}^{K}(P)=\beta_{h_{1}-i, h_{1}+d-j}^{K}(P) .
$$

This implies the remaining assertions.

Remark 2.5 Note that the conjecture of Kalai, Kleinschmidt, and Lee has been shown by giving a combinatorial interpretation of the first graded Betti numbers of a simplicial polytope. By duality, it follows that the second last non-trivial graded Betti numbers have a combinatorial interpretation, too. However, it is not possible to find combinatorial interpretations of all graded Betti numbers because, in general, the Betti numbers depend on the characteristic of the ground field (see Example 3.3 of [23]).

\section{Upper Bounds for Betti Numbers}

The key to proving the conjecture of Kalai, Kleinschmidt, and Lee has been to identify the number of missing $i$-simplices as a certain first graded Betti number. The results in [16] show that in order to compute an upper bound for this number in terms of the $h$-vector of the polytope, we need to know an upper bound for the Betti numbers of Cohen-Macaulay algebras. The goal of this section is to establish such bounds. Since the general case does not take more work than the special case of a Cohen-Macaulay algebra, we derive upper bounds for the graded Betti numbers of any arbitrary standard graded $K$-algebra in terms of its Hilbert function.

The applications in Sect. 4 rely on the results about the first graded Betti numbers of certain Gorenstein algebras. However, we cannot restrict ourselves to considering first Betti numbers in this section. In order to apply Theorem 8.13 in [16], we also need optimal bounds for the last non-trivial Betti numbers of a Cohen-Macaulay algebra. This forces us to discuss simultaneously all graded Betti numbers here.

Throughout this section we denote by $R$ the polynomial ring $K\left[x_{1}, \ldots, x_{n}\right]$ over an arbitrary field $K$ with its standard grading where every variable has degree one. $A \neq 0$ will be a standard graded $K$-algebra $R / I$ where $I \subset R$ is a proper homogeneous ideal. For a finitely generated graded $R$-module $M=\bigoplus_{j \in \mathbb{Z}}[M]_{j}$, we denote its graded Betti numbers by

$$
\beta_{i j}^{R}(M):=\operatorname{dim}_{K}\left[\operatorname{Tor}_{i}^{R}(M, K)\right]_{j} .
$$

Since the graded Betti numbers of $M$ do not change under field extensions of $K$, we may and will assume that the field $K$ is infinite.

The Hilbert function of $M$ is the numerical function $h_{M}: \mathbb{Z} \rightarrow \mathbb{Z}, h_{M}(j):=$ $\operatorname{dim}_{K}[M]_{j}$. The Hilbert functions of graded $K$-algebras have been completely classified by Macaulay. In order to state his result we need some notation. 
Notation 3.1 (i) We always use the following convention for binomial coefficients: If $a \in \mathbb{R}$ and $j \in \mathbb{Z}$, then

$$
\left(\begin{array}{l}
a \\
j
\end{array}\right):= \begin{cases}a(a-1) \cdots(a-j+1) / j ! & \text { if } j>0 \\
1 & \text { if } j=0 \\
0 & \text { if } j<0\end{cases}
$$

(ii) Let $b, d$ be positive integers. Then there are uniquely determined integers $m_{d}>$ $m_{d-1}>m_{s} \geq s \geq 1$ such that

$$
b=\left(\begin{array}{c}
m_{d} \\
d
\end{array}\right)+\left(\begin{array}{c}
m_{d-1} \\
d-1
\end{array}\right)+\cdots+\left(\begin{array}{c}
m_{s} \\
s
\end{array}\right) .
$$

This is called the $d$-binomial expansion of $b$. For any integer $j$ we set

$$
b^{\langle d, j\rangle}:=\left(\begin{array}{c}
m_{d}+j \\
d+j
\end{array}\right)+\left(\begin{array}{c}
m_{d-1}+j \\
d-1+j
\end{array}\right)+\cdots+\left(\begin{array}{c}
m_{s}+j \\
s+j
\end{array}\right) .
$$

Of particular importance are the cases where $j=1$ or $j=-1$. To simplify notation, we further define

$$
b^{\langle d\rangle}:=b^{\langle d, 1\rangle}=\left(\begin{array}{c}
m_{d}+1 \\
d+1
\end{array}\right)+\left(\begin{array}{c}
m_{d-1}+1 \\
d
\end{array}\right)+\cdots+\left(\begin{array}{c}
m_{s}+1 \\
s+1
\end{array}\right)
$$

and

$$
b_{[d]}:=b^{\langle d,-1\rangle}=\left(\begin{array}{c}
m_{d}-1 \\
d-1
\end{array}\right)+\left(\begin{array}{c}
m_{d-1}-1 \\
d-2
\end{array}\right)+\cdots+\left(\begin{array}{c}
m_{s}-1 \\
s-1
\end{array}\right) .
$$

(iii) If $b=0$, then we put $b^{\langle d\rangle}=b_{[d]}=b^{\langle d, j\rangle}:=0$ for all $j, d \in \mathbb{Z}$.

Recall that a sequence of non-negative integers $\left(h_{j}\right)_{j \geq 0}$ is called an $O$-sequence if $h_{0}=1$ and $h_{j+1} \leq h_{j}^{\langle j\rangle}$ for all $j \geq 1$. Now we can state Macaulay's characterization of Hilbert functions [12] (see also [19]).

Theorem 3.2 (Macaulay) For a numerical function $h: \mathbb{Z} \rightarrow \mathbb{Z}$, the following conditions are equivalent:

(a) $h$ is the Hilbert function of a standard graded $K$-algebra.

(b) $h(j)=0$ if $j<0$ and $\{h(j)\}_{j \geq 0}$ is an $O$-sequence.

For later use we record some formulas for sums involving binomial coefficients.

Lemma 3.3 For any positive real numbers $a, b$ and every integer $j \geq 0$, there are the following identities:

(i) $\sum_{k=0}^{j}(-1)^{k}\left(\begin{array}{c}a+k-1 \\ k\end{array}\right)\left(\begin{array}{c}b \\ j-k\end{array}\right)=\left(\begin{array}{c}b-a \\ j\end{array}\right)$; 
(ii) $\sum_{k=0}^{j}\left(\begin{array}{c}a+k-1 \\ k\end{array}\right)\left(\begin{array}{c}b+j-k-1 \\ j-k\end{array}\right)=\left(\begin{array}{c}a+b+j-1 \\ j\end{array}\right)$;

(iii) $\sum_{k=0}^{j}(-1)^{k}\left(\begin{array}{c}a+k \\ m\end{array}\right)\left(\begin{array}{c}b \\ j-k\end{array}\right)=\sum_{k=0}^{m}\left(\begin{array}{c}a-k-1 \\ m-k\end{array}\right)\left(\begin{array}{c}b-k-1 \\ j\end{array}\right) \begin{aligned} & \text { if } 0 \leq m \leq a \\ & \text { are integers. }\end{aligned}$

Proof (i) and (ii) are probably standard. In any case they follow immediately by comparing coefficients of power series using the identities $(1+x)^{b-a}=(1+x)^{-a}$. $(1+x)^{b}$ and $(1-x)^{-a-b}=(1-x)^{-a} \cdot(1-x)^{-b}$.

To see part (iii), we first use (ii) and finally (i); we get

$$
\begin{aligned}
\sum_{k=0}^{j}(-1)^{k}\left(\begin{array}{c}
a+k \\
m
\end{array}\right)\left(\begin{array}{c}
b \\
j-k
\end{array}\right) & =\sum_{k=0}^{j}(-1)^{k}\left(\begin{array}{c}
b \\
j-k
\end{array}\right)\left\{\sum_{i=0}^{m}\left(\begin{array}{c}
k+i \\
i
\end{array}\right)\left(\begin{array}{c}
a-1-i \\
m-i
\end{array}\right)\right\} \\
& =\sum_{i=0}^{m}\left(\begin{array}{c}
a-1-i \\
m-i
\end{array}\right)\left\{\sum_{k=0}^{j}(-1)^{k}\left(\begin{array}{c}
k+i \\
k
\end{array}\right)\left(\begin{array}{c}
b \\
j-k
\end{array}\right)\right\} \\
& =\sum_{k=0}^{m}\left(\begin{array}{c}
a-1-i \\
m-i
\end{array}\right)\left(\begin{array}{c}
b-i-1 \\
j
\end{array}\right),
\end{aligned}
$$

as claimed.

After these preliminaries we are ready to derive bounds for Betti numbers. We begin with the special case of modules having a $d$-linear resolution. Recall that the graded module $M$ is said to have a $d$-linear resolution if it has a graded minimal free resolution of the form

$$
\cdots \rightarrow R^{\beta_{i}}(-d-i) \rightarrow \cdots \rightarrow R^{\beta_{1}}(-d-1) \rightarrow R^{\beta_{0}}(-d) \rightarrow M \rightarrow 0 .
$$

Here $\beta_{i}^{R}(M)=\sum_{j \in \mathbb{Z}} \beta_{i, j}^{R}(M):=\beta_{i}$ is the $i$ th total Betti number of $M$.

Proposition 3.4 Let $M \neq 0$ be a graded $R$-module with a d-linear resolution. Then, for every $i \geq 0$, its $i$ th total graded Betti number is

$$
\beta_{i}^{R}(M)=\sum_{j=0}^{i}(-1)^{j} \cdot h_{M}(d+j) \cdot\left(\begin{array}{c}
n \\
i-j
\end{array}\right)
$$

Proof We argue by induction on $i$. The claim is clear if $i=0$. Let $i>0$. Using the additivity of vector space dimensions along exact sequences and the induction hypothesis we get

$$
\begin{aligned}
\beta_{i}^{R}(M) & =(-1)^{i} h_{M}(d+i)+\sum_{j=0}^{i-1}(-1)^{i-1-j} \cdot \beta_{j}^{R}(M)\left(\begin{array}{c}
n-1+i-j \\
i-j
\end{array}\right) \\
& =(-1)^{i} h_{M}(d+i)
\end{aligned}
$$




$$
\begin{aligned}
& +\sum_{j=0}^{i-1}(-1)^{i-1-j} \cdot\left(\begin{array}{c}
n-1+i-j \\
i-j
\end{array}\right)\left\{\sum_{k=0}^{j}(-1)^{j} \cdot h_{M}(d+k)\left(\begin{array}{c}
n \\
j-k
\end{array}\right)\right\} \\
= & (-1)^{i} h_{M}(d+i) \\
& +\sum_{k=0}^{i-1}(-1)^{k} \cdot h_{M}(d+k)\left\{\sum_{j=k}^{i-1}(-1)^{i-1-j}\left(\begin{array}{c}
n-1-i-j \\
i-j
\end{array}\right)\left(\begin{array}{c}
n \\
j-k
\end{array}\right)\right\} \\
= & (-1)^{i} h_{M}(d+i) \\
& +\sum_{k=0}^{i-1}(-1)^{k} \cdot h_{M}(d+k)\left\{\sum_{j=1}^{i-k}(-1)^{j-1}\left(\begin{array}{c}
n+j-1 \\
j
\end{array}\right)\left(\begin{array}{c}
n \\
i-k-j
\end{array}\right)\right\} \\
= & (-1)^{i} h_{M}(d+i)+\sum_{k=0}^{i-1}(-1)^{k} \cdot h_{M}(d+k)\left(\begin{array}{c}
n \\
i-k
\end{array}\right)
\end{aligned}
$$

according to Lemma 3.3(i). Now the claim follows.

It is amusing and useful to apply this result to a case where we know the graded Betti numbers.

Example 3.5 Consider the ideal $I=\left(x_{1}, \ldots, x_{n}\right)^{d}$, where $d>0$. Its minimal free resolution is given by an Eagon-Northcott complex. It has a $d$-linear resolution and its Betti numbers are (see, e.g., the proof of Corollary 8.14 of [16])

$$
\beta_{i}^{R}(I)=\left(\begin{array}{c}
d+i-1 \\
i
\end{array}\right)\left(\begin{array}{c}
n+d-1 \\
d+i
\end{array}\right) .
$$

Since the Hilbert function of $I$ is, for all $j \geq 0$,

$$
h_{I}(d+j)=h_{R}(d+j)=\left(\begin{array}{c}
n+d+j-1 \\
d+j
\end{array}\right),
$$

a comparison with Proposition 3.4 yields

$$
\left(\begin{array}{c}
d+i-1 \\
i
\end{array}\right)\left(\begin{array}{c}
n+d-1 \\
d+i
\end{array}\right)=\sum_{j=0}^{i}(-1)^{j} \cdot\left(\begin{array}{c}
n+d+j-1 \\
d+j
\end{array}\right)\left(\begin{array}{c}
n \\
i-j
\end{array}\right) .
$$

Now we compute the graded Betti numbers of lex-segment ideals. Recall that an ideal $I \subset R$ is called a lex-segment ideal if, for every $d$, the ideal $I_{\langle d\rangle}$ is generated by the first $\operatorname{dim}_{k}[I]_{d}$ monomials in the lexicographic order of the monomials in $R$. Here $I_{\langle d\rangle}$ is the ideal that is generated by all the polynomials of degree $d$ in $I$. For every graded $K$-algebra $A=R / I$ there is a unique lex-segment ideal $I^{\text {lex }} \subset R$ such that $A$ and $R / I^{\text {lex }}$ have the same Hilbert function. For further information on lex-segment ideals we refer to [3]. 
Lemma 3.6 Let $I \subset R$ be a proper lex-segment ideal whose generators all have degree $d$. Consider the $d$-binomial expansion of $b:=h_{R / I}(d)$ :

$$
b=\left(\begin{array}{c}
m_{d} \\
d
\end{array}\right)+\left(\begin{array}{c}
m_{d-1} \\
d-1
\end{array}\right)+\cdots+\left(\begin{array}{c}
m_{s} \\
s
\end{array}\right) .
$$

Then the Betti numbers of $A:=R / I$ are for all $i \geq 0$,

$$
\begin{aligned}
\beta_{i+1}^{R}(A) & =\beta_{i+1, i+d}^{R}(A) \\
& =\left(\begin{array}{c}
n+d-1 \\
d+i
\end{array}\right)\left(\begin{array}{c}
d+i-1 \\
d-1
\end{array}\right)-\sum_{k=s}^{d} \sum_{j=0}^{m_{k}-k}\left(\begin{array}{c}
m_{k}-j-1 \\
k-1
\end{array}\right)\left(\begin{array}{c}
n-1-j \\
i
\end{array}\right) .
\end{aligned}
$$

(Note that according to Notation 3.1, the sum on the right-hand side is zero if $b=0$.)

Proof Gotzmann's Persistence Theorem [4] implies that the Hilbert function of $A$ is, for $j \geq 0, h_{A}(d+j)=b^{\langle d, j\rangle}$ and that $I$ has a $d$-linear resolution. Hence Proposition 3.4 in conjunction with formula (3.1) and Lemma 3.3(iii) provides

$$
\begin{aligned}
\beta_{i+1}^{R}(A) & =\beta_{i}^{R}(I)=\sum_{j=0}^{i}(-1)^{j} \cdot h_{I}(d+j)\left(\begin{array}{c}
n \\
i-j
\end{array}\right) \\
& =\sum_{j=0}^{i}(-1)^{j}\left[\left(\begin{array}{c}
n+d+j-1 \\
d+j
\end{array}\right)-b^{\langle d, j\rangle}\right]\left(\begin{array}{c}
n \\
i-j
\end{array}\right) \\
& =\left(\begin{array}{c}
n+d-1 \\
d+i
\end{array}\right)\left(\begin{array}{c}
d+i-1 \\
i
\end{array}\right)-\sum_{j=0}^{i}(-1)^{j} \cdot\left[\sum_{k=s}^{d}\left(\begin{array}{c}
m_{k}+j \\
k+j
\end{array}\right)\right]\left(\begin{array}{c}
n \\
i-j
\end{array}\right) \\
& =\left(\begin{array}{c}
n+d-1 \\
d+i
\end{array}\right)\left(\begin{array}{c}
d+i-1 \\
i
\end{array}\right)-\sum_{k=s}^{d}\left[\sum_{j=0}^{i}(-1)^{j} \cdot\left(\begin{array}{c}
m_{k}+j \\
m_{k}-k
\end{array}\right)\left(\begin{array}{c}
n \\
i-j
\end{array}\right)\right] \\
& =\left(\begin{array}{c}
n+d-1 \\
d+i
\end{array}\right)\left(\begin{array}{c}
d+i-1 \\
i
\end{array}\right)-\sum_{k=s}^{d} \sum_{j=0}^{m_{k}-k}\left(\begin{array}{c}
m_{k}-j-1 \\
k-1
\end{array}\right)\left(\begin{array}{c}
n-1-j \\
i
\end{array}\right),
\end{aligned}
$$

as claimed.

The above formulas simplify in the extremal cases.

Corollary 3.7 Adopt the notation and assumptions of Lemma 3.6. Then

(a) $\beta_{1}^{R}(A)=\left(\begin{array}{c}n+d-1 \\ d\end{array}\right)-b$

(b) $\beta_{n}^{R}(A)=\left(\begin{array}{c}n+d-2 \\ d-1\end{array}\right)-b_{[d]}$. 
Proof Part (a) being clear, we restrict ourselves to showing (b). Since $\left(\begin{array}{c}n-1-j \\ n-1\end{array}\right)=0$ for $j>0$, Lemma 3.6 immediately gives $\beta_{n}^{R}(A)=\left(\begin{array}{c}n+d-2 \\ d-1\end{array}\right)-\sum_{k=s}^{d}\left(\begin{array}{c}m_{k}-1 \\ k-1\end{array}\right)=$ $\left(\begin{array}{c}n+d-2 \\ d-1\end{array}\right)-b_{[d]}$.

Now, we can compute the non-trivial graded Betti numbers of an arbitrary lexsegment ideal. The basic idea is to reduce the computation to the special case treated in Lemma 3.6 by exploiting algebraic properties of lex-segment ideals.

Proposition 3.8 Let $I \subset R$ be an arbitrary proper lex-segment ideal and let $d \geq 2$ be an integer. Set $A:=R / I$ and consider the d-binomial expansion

$$
h_{A}(d)=:\left(\begin{array}{c}
m_{d} \\
d
\end{array}\right)+\left(\begin{array}{c}
m_{d-1} \\
d-1
\end{array}\right)+\cdots+\left(\begin{array}{c}
m_{s} \\
s
\end{array}\right)
$$

and the $(d-1)$-binomial expansion

$$
h_{A}(d-1)=:\left(\begin{array}{l}
n_{d-1} \\
d-1
\end{array}\right)+\left(\begin{array}{c}
n_{d-2} \\
d-2
\end{array}\right)+\cdots+\left(\begin{array}{c}
n_{t} \\
t
\end{array}\right) .
$$

Then we have for all $i \geq 0$

$$
\beta_{i+1, i+d}^{R}(A)=\beta_{i+1, i+d}\left(h_{A}, n\right),
$$

where

$$
\begin{aligned}
\beta_{i+1, i+d}\left(h_{A}, n\right):= & \sum_{k=t}^{d-1} \sum_{j=0}^{n_{k}-k}\left(\begin{array}{c}
n_{k}-j \\
k
\end{array}\right)\left(\begin{array}{c}
n-1-j \\
i
\end{array}\right) \\
& -\sum_{k=s}^{d} \sum_{j=0}^{m_{k}-k}\left(\begin{array}{c}
m_{k}-1-j \\
k-1
\end{array}\right)\left(\begin{array}{c}
n-1-j \\
i
\end{array}\right) .
\end{aligned}
$$

Proof As noted above, since $I$ is a lex-segment ideal, for every $j \in \mathbb{Z}$, the ideal $I_{\langle j\rangle}$ has a $j$-linear resolution, i.e., the ideal $I$ is componentwise linear. Hence, Proposition 1.3 of [7] gives for all $i \geq 0$,

$$
\beta_{i+1, i+d}^{R}(A)=\beta_{i+1}^{R}\left(R / I_{\langle d\rangle}\right)-\beta_{i+1}^{R}\left(R / \mathfrak{m} I_{\langle d-1\rangle}\right),
$$

where $\mathfrak{m}=\left(x_{1}, \ldots, x_{n}\right)$ is the homogeneous maximal ideal of $R$.

Since $I_{\langle d-1\rangle}$ is generated in degree $d-1$, the ideals $I_{\langle d-1\rangle}$ and $\mathfrak{m} I_{\langle d-1\rangle}$ have the same Hilbert function in all degrees $j \geq d$. Thus, using the assumption $d \geq 2$, Gotzmann's Persistence Theorem [4] provides

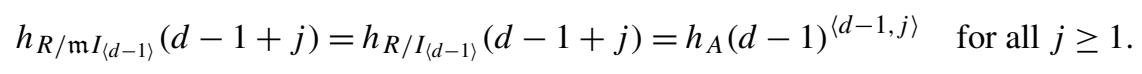

It is easy to see that $\mathfrak{m} I_{\langle d-1\rangle}$ has a $d$-linear resolution because $I_{\langle d-1\rangle}$ has a $(d-1)$ linear resolution. Hence, as in the proof of Lemma 3.6, Proposition 3.4 provides

$$
\beta_{i+1}^{R}\left(R / \mathfrak{m} I_{\langle d-1\rangle}\right)=\left(\begin{array}{c}
n+d-1 \\
d+i
\end{array}\right)\left(\begin{array}{c}
d+i-1 \\
d-1
\end{array}\right)-\sum_{k=t}^{d-1} \sum_{j=0}^{n_{k}-k}\left(\begin{array}{c}
n_{k}-j \\
k
\end{array}\right)\left(\begin{array}{c}
n-1-j \\
i
\end{array}\right) .
$$


Plugging this and the result of Lemma 3.6 into the formula (3.2), we get our claim.

Again, the formula simplifies in the extremal cases. We use the result in the following section.

Corollary 3.9 Adopt the notation and assumptions of Proposition 3.8. Then:

(a) $\beta_{1, d}^{R}(A)=\beta_{1, d}\left(h_{A}, n\right)=h_{A}(d-1)^{\langle d-1\rangle}-h_{A}(d)$.

(b) $\beta_{n, n-1+d}^{R}(A)=\beta_{n, n-1+d}\left(h_{A}, n\right)=h_{A}(d-1)-\left(h_{A}(d)\right)_{[d]}$.

Proof This follows from the formula given in Proposition 3.8.

In Proposition 3.8 we left out the case $d \leq 1$ which is easy to deal with. We need:

Definition 3.10 Let $h$ be the Hilbert function of a graded $K$-algebra such that $h(1) \leq n$. Then we define, for all integers $i \geq 0$ and $d$, the numbers $\beta_{i+1, i+d}(h, n)$ as in Proposition 3.8 if $d \geq 2$ and otherwise:

$$
\beta_{i+1, i+d}(h, n):= \begin{cases}\left(\begin{array}{c}
n-h(1) \\
i+1
\end{array}\right) & \text { if } d=1 \\
0 & \text { if } d \leq 0\end{cases}
$$

Moreover, if $i \leq 0$ we set

$$
\beta_{i, j}(h, n):= \begin{cases}1 & \text { if }(i, j)=(0,0) \\ 0 & \text { otherwise }\end{cases}
$$

Lemma 3.11 Let $A=R / I \neq 0$ be any graded $K$-algebra. Then we have for all integers $i, d$ with $d \leq 1$,

$$
\beta_{i+1, i+d}^{R}(A)=\beta_{i+1, i+d}\left(h_{A}, n\right) .
$$

Proof Since $A$ has as an $R$-module just one generator in degree zero, this is clear if $d \leq 0$. Furthermore, $I_{\langle 1\rangle}$ is generated by a regular sequence of length $n-h_{A}(1)$. Its minimal free resolution is given by the Koszul complex. Hence, the claim follows for $d=1$ because $\beta_{i+1, i+1}^{R}(A)=\beta_{i, i+1}^{R}\left(I_{\langle 1\rangle}\right)$.

Combined with results of Bigatti, Hullet, and Pardue, we get the main result of this section: bounds for the graded Betti numbers of a $K$-algebra as an $R$-module in terms of its Hilbert function and the dimension of $R$.

Theorem 3.12 Let $A=R / I \neq 0$ be a graded $K$-algebra. Then its graded Betti numbers are bounded by

$$
\beta_{i+1, i+j}^{R}(A) \leq \beta_{i+1, i+j}\left(h_{A}, n\right) \quad(i, j \in \mathbb{Z}) .
$$

Furthermore, equality is attained for all integers $i, j$ if I is a lex-segment ideal. 
Proof Let $I^{\text {lex }} \subset R$ be the lex-segment ideal such that $A$ and $R / I^{\text {lex }}$ have the same Hilbert function. Then we have for all integers $i, j$ that

$$
\beta_{i+1, i+j}^{R}(A) \leq \beta_{i+1, i+j}^{R}\left(R / I^{\mathrm{lex}}\right)
$$

according to Bigatti [1] and Hulett [8] if char $K=0$ and to Pardue [17] if $K$ has positive characteristic. Since Proposition 3.8 and Lemma 3.11 yield

$$
\beta_{i+1, i+j}^{R}\left(R / I^{\text {lex }}\right)=\beta_{i+1, i+j}\left(h_{A}, n\right) \quad(i, j \in \mathbb{Z}),
$$

our claims follow.

Remark 3.13 Note that Theorem 3.12 gives in particular that $\beta_{i+1, i+d}^{R}(A)=0$ if $i \geq n$, in accordance with Hilbert's Syzygy Theorem.

We conclude this section by discussing the graded Betti numbers of CohenMacaulay algebras with the so-called Weak Lefschetz property. The special case of a Gorenstein algebra is crucial for the applications to polytopes in the following section.

Let $A=R / I$ be a graded Cohen-Macaulay $K$-algebra of Krull dimension $d$ and let $l_{1}, \ldots, l_{d} \in[R]_{1}$ be sufficiently general linear forms. Then $\bar{A}:=A /\left(l_{1}, \ldots, l_{d}\right) A$ is called the Artinian reduction of $A$. Its Hilbert function and graded Betti numbers as a module over $\bar{R}:=R /\left(l_{1}, \ldots, l_{d}\right) R$ do not depend on the choice of the forms $l_{1}, \ldots, l_{d}$. The Hilbert function of $\bar{A}$ takes positive values in only finitely many degrees. The sequence of these positive integers $\underline{h}=\left(h_{0}, h_{1}, \ldots, h_{r}\right)$ is called the $h$-vector of $A$. We set $\beta_{i+1, i+d}(\underline{h}, n-d):=\beta_{i+1, i+d}\left(h_{\bar{A}}, n-d\right)$. Using this notation we get:

Corollary 3.14 Let $A=R / I$ be a Cohen-Macaulay graded $K$-algebra of dimension $d$ with h-vector $\underline{h}$. Then its graded Betti numbers satisfy

$$
\beta_{i+1, i+j}^{R}(A) \leq \beta_{i+1, i+j}(\underline{h}, n-d) \quad(i, j \in \mathbb{Z}) .
$$

Proof If $l \in[R]_{1}$ is a not a zero-divisor of $A$, then the graded Betti numbers of $A$ as an $R$-module agree with the graded Betti numbers of $A / l A$ as an $R / l R$ module (see, e.g., Corollary 8.5 of [16]). Hence, by passing to the Artinian reduction of $A$, Theorem 3.12 provides the claim.

Remark 3.15 Note that, for any O-sequence $\underline{h}=\left(1, h_{1}, \ldots, h_{r}\right)$ with $h_{r}>0$, Definition 3.10 provides $\beta_{i+1, i+j}(\underline{h}, m)=0$ for all $i, m \geq 0$ if $j \leq 1$ or $j \geq r+2$.

Recall that an Artinian graded $K$-algebra $A$ has the so-called Weak Lefschetz property if there is an element $l \in A$ of degree one such that, for each $j \in \mathbb{Z}$, the multiplication $\times l:[A]_{j-1} \rightarrow[A]_{j}$ has maximal rank. The Cohen-Macaulay $K$-algebra $A$ is said to have the Weak Lefschetz property if its Artinian reduction has the Weak Lefschetz property. 
Remark 3.16 The Hilbert functions of Cohen-Macaulay algebras with the Weak Lefschetz property have been completely classified in Proposition 3.5 of [6]. Moreover, Theorem 3.20 in [6] gives optimal upper bounds on their graded Betti numbers in terms of the Betti numbers of certain lex-segment ideals. Thus, combining this result with Theorem 3.12, one gets upper bounds for the Betti numbers of these algebras in terms of their Hilbert functions. In general, these bounds are strictly smaller than the bounds of Corollary 3.14 for Cohen-Macaulay algebras that do not necessarily have the Weak Lefschetz property.

The $h$-vectors of graded Gorenstein algebras with the Weak Lefschetz property are precisely the SI-sequences (see Theorem 6.3 of [16] or Theorem 1.2 of [5]). For their Betti numbers we obtain:

Theorem 3.17 Let $\underline{h}=\left(1, h_{1}, \ldots, h_{u}, \ldots, h_{r}\right)$ be an SI-sequence where $h_{u-1}<$ $h_{u}=\cdots=h_{r-u}>h_{r-u+1}$. Put $g=\left(1, h_{1}-1, h_{2}-h_{1}, \ldots, h_{u}-h_{u-1}\right)$. If $A=R / I$ is a Gorenstein graded $K$-algebra of dimension $d$ with the Weak Lefschetz property and $h$-vector $\underline{h}$, then its graded Betti numbers satisfy

$\beta_{i+1, i+j}^{R}(A) \leq \begin{cases}\beta_{i+1, i+j}(\underline{g}, m) & \text { if } j \leq r-u, \\ \beta_{i+1, i+j}(\underline{g}, m)+\beta_{g_{1}-i, r+h_{1}-i-j}(\underline{g}, m) & \text { if } r-u+1 \leq j \leq u+1, \\ \beta_{g_{1}-i, r+h_{1}-i-j}(\underline{g}, m) & \text { if } j \geq u+2,\end{cases}$

where $m:=n-d-1=\operatorname{dim} R-d-1$.

Proof This follows immediately by combining Theorem 8.13 of [16] and Theorem 3.12.

\section{Explicit Bounds for the Number of Missing Simplices}

We now return to the consideration of simplicial polytopes. To this end we specialize the results of Sect. 3 and then discuss some applications.

We begin by simplifying our notation somewhat. Let $P$ be a simplicial $d$-polytope with $f$-vector $f$. It is well known that the $h$-vector of the Stanley-Reisner ring $K[P]$ agrees with the $h$-vector of $P$ as defined in the Introduction. Furthermore, in Sect. 2 we defined the graded Betti numbers of $K[P]=R / I_{P}$ by resolving $K[P]$ as an $R$ module where $R$ is a polynomial ring of dimension $f_{0}$ over $K$, i.e.,

$$
\beta_{i, j}^{K}(P)=\beta_{i, j}^{R}(K[P]) .
$$

Note that the Stanley-Reisner ideal $I_{P}$ does not contain any linear forms. The graded Betti numbers of $P$ agree with the graded Betti numbers of the Artinian reduction of $K[P]$ as a module over a polynomial ring of dimension $f_{0}-d=h_{1}$. Thus, we can simplify the statements of the bounds of $\beta_{i, j}^{K}(P)$ by setting:

Notation 4.1 Using the notation introduced above Corollary 3.14 we define for every O-sequence $\underline{h}$,

$$
\beta_{i+1, i+j}(\underline{h}):=\beta_{i+1, i+j}\left(\underline{h}, h_{1}\right) .
$$


Notice that $\beta_{i+1, i+j}(\underline{h})=0$ if $i \geq 0$ and $j \leq 1$.

In this section we primarily use the $g$-vector of a polytope which is defined as follows:

Definition 4.2 Let $P$ be a simplicial polytope with $h$-vector $\underline{h}:=\left(h_{0}, \ldots, h_{d}\right)$. Then the g-Theorem (Theorem 1.1) shows that there is a unique integer $u$ such that $h_{u-1}<h_{u}=\cdots=h_{d-u}>h_{d-u+1}$. The vector $g=\left(g_{0}, \ldots, g_{u}\right):=\left(1, h_{1}-1, h_{2}-\right.$ $\left.h_{1}, \ldots, h_{u}-h_{u-1}\right)$ is called the $g$-vector of $P$. $\overline{\text { All }}$ its entries are positive.

Some observations are in order.

Remark 4.3 (i) By its definition, the $g$-vector of the polytope $P$ is uniquely determined by the $h$-vector of $P$. The g-Theorem shows that the $h$-vector of $P$ (thus also its $f$-vector) can be recovered from its $g$-vector, provided the dimension of $P$ is given.

(ii) The g-Theorem also gives an estimate of the length of the $g$-vector because it implies $2 u \leq d=\operatorname{dim} P$.

Now we can state our explicit bounds for the Betti numbers of a polytope.

Theorem 4.4 Let $K$ be a field of characteristic zero and let $\underline{g}=\left(g_{0}, \ldots, g_{u}\right)$ be an $O$-sequence with $g_{u}>0$. Then we have:

(a) If $P$ is a simplicial d-polytope with g-vector $\underline{g}$, then

$$
\beta_{i+1, i+j}^{K}(P) \leq \begin{cases}\beta_{i+1, i+j}(\underline{g}) & \text { if } j \leq d-u, \\ \left.\beta_{i+1, i+j} \underline{(g}\right)+\beta_{g_{1}-i, d+h_{1}-i-j}(\underline{g}) & \text { if } d-u+1 \leq j \leq u+1, \\ \beta_{g_{1}-i, d+g_{1}+1-i-j(\underline{g})} & \text { if } j \geq u+2 .\end{cases}
$$

(b) In (a) equality is attained for all integers $i, j$ if $P$ is the d-dimensional BilleraLee polytope with g-vector $g$.

Proof It is well known that the Stanley-Reisner ring of every simplicial polytope is a Gorenstein algebra. Furthermore, according to Stanley [20] (see also [15]), it has the Weak Lefschetz property. Hence part (a) is a consequence of Theorem 3.17. Part (b) follows from Theorem 9.6 of [16] and Theorem 3.12, as pointed out in the proof of Theorem 2.1.

We have seen in Sect. 2 that the number of empty $j$-simplices of the simplicial polytope $P$ is equal to the Betti number $\beta_{1, j+1}^{K}(P)$. Thus, we want to make the preceding bounds more explicit if $i=0$. At first, we treat a trivial case.

Remark 4.5 Notice that the $g$-vector has length one, i.e., $u=0$ if and only if the polytope $P$ is a simplex. In this case, its Stanley-Reisner ideal is a principal ideal generated by a monomial of degree $d=\operatorname{dim} P$.

In the following result we stress when the Betti numbers vanish. Because of Remark 4.5, it is harmless to assume that $u \geq 1$. We use Notation 3.1. 
Corollary 4.6 Let $\underline{g}=\left(g_{0}, \ldots, g_{u}\right)$ be an O-sequence with $g_{u}>0$ and $u \geq 1$. Set $g_{u+1}:=0$. Then we have:

(a) If $P$ is a simplicial d-polytope with $g$-vector $g$, then there are the following bounds:

(i) If $d \geq 2 u+1$, then

$$
\beta_{1, j}^{K}(P) \leq \begin{cases}g_{j-1}^{\langle j-1\rangle}-g_{j} & \text { if } 2 \leq j \leq u+1, \\ \left.g_{d+1-j}-\left(g_{d+2-j}\right)_{[d+2-j]}\right] & \text { if } d-u+1 \leq j \leq d, \\ 0 & \text { otherwise. }\end{cases}
$$

(ii) If $d=2 u$, then

$$
\beta_{1, j}^{K}(P) \leq \begin{cases}g_{j-1}^{\langle j-1\rangle}-g_{j} & \text { if } 2 \leq j \leq u, \\ g_{u}^{\langle u\rangle}+g_{u} & \text { if } j=u+1, \\ \left.g_{d+1-j}-\left(g_{d+2-j}\right)_{[d+2-j]}\right] & \text { if } u+2 \leq j \leq d, \\ 0 & \text { otherwise. }\end{cases}
$$

(b) In (a) equality is attained for all integers $j$ if $P$ is the d-dimensional Billera-Lee polytope with g-vector $\underline{g}$.

Proof Since the first Betti numbers of any polytope do not depend on the characteristic of the field, the claims follow from Theorem 4.4 by taking into account Corollary 2.4, Corollary 3.9, and the fact that $\beta_{i+1, i+j}(\underline{g})=0$ if $i \geq 0$ and either $j \leq 1$ or $j \geq u+2$ due to Remark 3.15.

To illustrate the last result, we consider an easy case.

Example 4.7 Let $P$ be a simplicial $d$-polytope with $g_{1}=1$. Then its Stanley-Reisner ideal $I_{P}$ is a Gorenstein ideal of height two, thus a complete intersection. Indeed, since the $g$-vector of $P$ is an $\mathrm{O}$-sequence, it must be $g=\left(g_{0}, \ldots, g_{u}\right)=(1, \ldots, 1)$. Hence Corollary 4.6 provides that $I_{P}$ has exactly two minimal generators, one of degree $u+1$ and one of degree $d-u+1$. Equivalently, $P$ has exactly two empty simplices, one of dimension $u$ and one of dimension $d-u$.

As an immediate consequence of Corollary 4.6 we partially recover Proposition 3.6 of [10].

Corollary 4.8 Every simplicial d-polytope has no empty faces of dimension $j$ if $u+1 \leq j \leq d-u-1$.

Remark 4.9 Kalai's Conjecture 8 in [10] states that the following converse of Corollary 4.8 should be true: If there is an integer $k$ such that $d \geq 2 k$ and the simplicial $d$-polytope has no empty simplices of dimension $j$ whenever $k \leq j \leq d-k$, then $u<k$. Kalai has proved this if $k=2$ in [9]. Our results provide the following weaker version of Kalai's conjecture: 
If there is an integer $k$ such that $d \geq 2 k$ and every simplicial $d$-polytope with $g$-vector $\left(g_{0}, \ldots, g_{u}\right)$ has no empty simplices of dimension $j$ whenever $k \leq j \leq d-k$, then $u<k$.

Indeed, this follows by the sharpness of the bounds in Corollary 4.6.

Now we want to make some existence results of Kalai and Perles effective. As preparation, we state:

Corollary 4.10 Let $P$ be a simplicial $d$-polytope with $g$-vector $g=\left(g_{0}, \ldots, g_{u}\right)$ where $u \geq 1$. Set $g_{u+1}=0$. Then the number $N(k)$ of empty simplices of $P$ whose dimension is at most $k$ is bounded above as follows:

$$
N(k) \leq \begin{cases}g_{1}+\sum_{j=1}^{k}\left\{g_{j}^{\langle j\rangle}-g_{j}\right\}-g_{k+1} & \text { if } 1 \leq k \leq \min \{u, d-u-1\}, \\ N(u) & \text { if } u<k<d-u, \\ g_{1}+g_{d-k}^{\langle d-k\rangle}+\sum_{j=1}^{d-k-1}\left\{g_{j}^{\langle j\rangle}-g_{j}\right\} & \\ \quad+\sum_{j=d-k+1}^{u}\left\{g_{j}^{\langle j\rangle}-\left(g_{j}\right)_{[j]}\right\} & \text { if } d-u \leq k<d .\end{cases}
$$

Furthermore, for each $k$, the bound is attained if $P$ is the Billera-Lee d-polytope with $g$-vector $g$.

Proof By Corollary 4.8, this is clear if $u<k<d-u$. In any case, we know that $N(k)=\sum_{j=2}^{k+1} \beta_{1, j}^{K}(P)$. Thus, using Corollary 4.6 carefully, elementary calculations provide the claim. We omit the details.

The last result immediately gives:

Corollary 4.11 If $P$ is a simplicial polytope with $g$-vector $g=\left(g_{0}, \ldots, g_{u}\right)$, where $u \geq 1$, then its total number of empty simplices is at most

$$
\left(\begin{array}{c}
g_{1}+2 \\
2
\end{array}\right)-1+\sum_{j=2}^{u}\left\{g_{j}^{\langle j\rangle}-\left(g_{j}\right)_{[j]}\right\} .
$$

Furthermore, this bound is attained if $P$ is any Billera-Lee polytope with g-vector $\underline{g}$.

Proof Use Corollary 4.10 with $k=d-1$ and recall that $g_{1}^{\langle 1\rangle}=\left(\begin{array}{c}g_{1}+1 \\ 2\end{array}\right)$.

Remark 4.12 It is somewhat surprising that the bound in Corollary 4.11 does not depend on the dimension of the polytope. In contrast, the other bounds (see, e.g., Corollary 4.10) do depend on the dimension $d$ of the polytope.

In view of Corollary 4.10, the following elementary facts will be useful. 
Lemma 4.13 Let $k$ be a positive integer. If $a \geq b$ are non-negative integers, then

(a) $a^{\langle k\rangle}-a_{[k]} \geq b^{\langle k\rangle}-b_{[k]}$;

(b) $a^{\langle k\rangle}-a \geq b^{\langle k\rangle}-b$;

(c) $a_{[k]} \geq b_{[k]}$.

Proof We show only (a). The proofs of the other claims are similar and easier.

To see (a), we begin by noting, for integers $m \geq j>0$, the identity

$$
\left(\begin{array}{c}
m+1 \\
j+1
\end{array}\right)-\left(\begin{array}{c}
m-1 \\
j-1
\end{array}\right)=\left(\begin{array}{c}
m \\
j+1
\end{array}\right)+\left(\begin{array}{c}
m-1 \\
j
\end{array}\right) .
$$

Now we use induction on $k \geq 1$. Since $a^{\langle 1\rangle}-a_{[1]}=\left(\begin{array}{c}a+1 \\ 2\end{array}\right)-1$, the claim is clear if $k=1$. Let $k \geq 2$. Consider the $k$-binomial expansions

$$
a=:\left(\begin{array}{c}
m_{k} \\
k
\end{array}\right)+\left(\begin{array}{c}
m_{k-1} \\
k-1
\end{array}\right)+\cdots+\left(\begin{array}{c}
m_{s} \\
s
\end{array}\right)
$$

and

$$
b=:\left(\begin{array}{c}
n_{k} \\
k
\end{array}\right)+\left(\begin{array}{c}
n_{k-1} \\
k-1
\end{array}\right)+\cdots+\left(\begin{array}{c}
n_{t} \\
t
\end{array}\right) .
$$

Since $a \geq b$, we get $m_{k} \geq n_{k}$. We distinguish two cases.

Case 1 . Let $m_{k}=n_{k}$. Then the claim follows by applying the induction hypothesis to

$$
a-\left(\begin{array}{c}
m_{k} \\
k
\end{array}\right) \geq b-\left(\begin{array}{c}
m_{k} \\
k
\end{array}\right) \text {. }
$$

Case 2. Let $m_{k}>n_{k}$. Using $n_{i} \leq n_{k}-k+i$ and formula (4.1), we get

$$
\begin{aligned}
b^{\langle k\rangle}-b_{[k]} & =\sum_{i=t}^{k}\left\{\left(\begin{array}{c}
n_{i} \\
i+1
\end{array}\right)+\left(\begin{array}{c}
n_{i}-1 \\
i
\end{array}\right)\right\} \\
& \leq \sum_{i=1}^{k}\left\{\left(\begin{array}{c}
n_{k}-k+i \\
i+1
\end{array}\right)+\left(\begin{array}{c}
n_{k}-k-1+i \\
i
\end{array}\right)\right\} \\
& =\left(\begin{array}{c}
n_{k}+1 \\
k+1
\end{array}\right)+\left(\begin{array}{c}
n_{k} \\
k
\end{array}\right)-\left(n_{k}-k+2\right) \\
& <\left(\begin{array}{c}
m_{k} \\
k+1
\end{array}\right)+\left(\begin{array}{c}
m_{k}-1 \\
k
\end{array}\right)
\end{aligned}
$$

because $n_{k}<m_{k}$. The claim follows since formula (4.1) gives $\left(\begin{array}{c}m_{k} \\ k+1\end{array}\right)+\left(\begin{array}{c}m_{k}-1 \\ k\end{array}\right) \leq$ $a^{\langle k\rangle}-a_{[k]}$.

Remark 4.14 In general, it is not true that $a>b$ implies $a^{\langle k\rangle}-a_{[k]}>b^{\langle k\rangle}-b_{[k]}$. For example, if $k \geq 2$ and $a-1=b=\left(\begin{array}{c}m \\ k\end{array}\right)>0$, then $a^{\langle k\rangle}-a_{[k]}=b^{\langle k\rangle}-b_{[k]}$. 
We are ready to establish optimal bounds that depend only on the dimension and the number of vertices.

Theorem 4.15 Let $P$ be a simplicial d-polytope with $d+g_{1}+1$ vertices which is not a simplex. Then there is the following bound on the number $N(k)$ of empty simplices of $P$ whose dimension is $\leq k$ :

$$
N(k) \leq \begin{cases}\left(\begin{array}{l}
g_{1}+k \\
g_{1}-1
\end{array}\right) & \text { if } 1 \leq k<d / 2 ; \\
\left(\begin{array}{c}
g_{1}+\lfloor d / 2\rfloor \\
g_{1}-1
\end{array}\right)+\left(\begin{array}{c}
g_{1}+\lfloor d / 2\rfloor-1 \\
g_{1}-1
\end{array}\right) & \text { if } d / 2 \leq k<d .\end{cases}
$$

Furthermore, for each $k$, the bound is attained if $P$ is the Billera-Lee d-polytope with $g$-vector $\left(g_{0}, \ldots, g_{u}\right)$ where $g_{j}=\left(\begin{array}{c}g_{1}+j-1 \\ j\end{array}\right), 0 \leq j \leq u$, and $u=\min \{k,\lfloor d / 2\rfloor\}$.

Proof Let $g=\left(g_{0}, \ldots, g_{u}\right)$ be the $g$-vector of $P$. Since $P$ is not a simplex, we have $u \geq 1$. We have to distinguish two cases.

Case 1 . Let $k<d / 2$. If $k>u$, then we formally set $g_{u+1}=\cdots=g_{\lfloor d / 2\rfloor}=0$. Since $k<d / 2 \leq d-u$, Corollary 4.10 provides

$$
N(k) \leq g_{1}+\sum_{j=1}^{k}\left\{g_{j}^{\langle j\rangle}-g_{j}\right\}-g_{k+1} .
$$

According to Lemma 4.13, the sum on the right-hand side becomes maximal if $g_{2}, \ldots, g_{k}$ are as large as possible and $g_{k+1}=0$. The latter means $u=k$. Macaulay's Theorem 3.2 implies $g_{j} \leq\left(\begin{array}{c}g_{1}+j-1 \\ j\end{array}\right)$. Now an easy computation provides the bound in this case. It is sharp because $\left(g_{0}, \ldots, g_{k}\right)$, where $g_{j}=\left(\begin{array}{c}g_{1}+j-1 \\ j\end{array}\right)$, is a $g$-vector of a simplicial $d$-polytope by the g-Theorem, thus Corollary 4.10 applies.

Case 2. Let $d / 2 \leq k<d$. First, we also assume that $k \geq d-u$. Then Corollary 4.10 gives

$$
N(k) \leq g_{1}+g_{d-k}^{\langle d-k\rangle}+\sum_{j=1}^{d-k-1}\left\{g_{j}^{\langle j\rangle}-g_{j}\right\}+\sum_{j=d-k+1}^{u}\left\{g_{j}^{\langle j\rangle}-\left(g_{j}\right)_{[j]}\right\} .
$$

Again, Lemma 4.13 shows that, for fixed $u$, the bound is maximized if $g_{j}=\left(\begin{array}{c}g_{1}+j-1 \\ j\end{array}\right)$, $0 \leq j \leq u$. This provides

$$
N(k) \leq\left(\begin{array}{c}
g_{1}+u \\
g_{1}-1
\end{array}\right)+\left(\begin{array}{c}
g_{1}+u-1 \\
g_{1}-1
\end{array}\right) .
$$

Since $u \leq d / 2$, our bound follows in this case.

Second, assume $k<d-u$. Then $u \leq d / 2 \leq k<d-u$ yields $u<d / 2$. Thus Corollary 4.10 provides $N(k)=N(u)$, but $N(u) \leq\left(\begin{array}{l}g_{1}+u \\ g_{1}-1\end{array}\right)$ by Case 1 . This concludes the proof of the bound in Case 2. Its sharpness is shown as in Case 1.

As an immediate consequence we obtain: 
Corollary 4.16 Every simplicial polytope, which is not a simplex, has at most $\left(\begin{array}{l}g_{1}+k \\ g_{1}-1\end{array}\right)+\left(\begin{array}{c}g_{1}+k-1 \\ g_{1}-1\end{array}\right)$ empty simplices of dimension $\leq k$.

Remark 4.17 Kalai [10, Theorem 2.7] has first given an estimate as in Corollary 4.16. His bound is

$$
N(k) \leq\left(g_{1}+1\right)^{k+1} \cdot(k+1) ! .
$$

Comparing with our bound, we see that Kalai's bound is asymptotically not optimal for $g_{1} \gg 0$.

Notice that the bound on $N(k)$ in Theorem 4.15 does not depend on $k$ if $k \geq d / 2$. This becomes plausible by considering cyclic polytopes.

Example 4.18 (i) Recall that a cyclic polytope $C\left(f_{0}, d\right)$ is a $d$-dimensional simplicial polytope which is the convex hull of $f_{0}$ distinct points on the moment curve

$$
\left\{\left(t, t^{2}, \ldots, t^{d}\right) \mid t \in \mathbb{R}\right\} .
$$

Its combinatorial type depends only on $f_{0}$ and $d$.

According to McMullen's Upper Bound Theorem [13], the cyclic polytope $C\left(f_{0}, d\right)$ has the maximal $f$-vector among all simplicial $d$-polytopes with $f_{0}$ vertices. Theorem 4.15 shows that it also has the maximal total number of empty simplices among these polytopes. Indeed, this follows by comparing with the main result in [22] (see also Corollary 9.10 of [16]) which provides that $C\left(f_{0}, d\right)$ has $\left(\begin{array}{c}g_{1}+\lfloor d / 2\rfloor \\ g_{1}-1\end{array}\right)+$ $\left(\begin{array}{c}g_{1}+\lfloor d / 2\rfloor-1 \\ g_{1}-1\end{array}\right)$ empty simplices. Moreover, the empty simplices of $C\left(f_{0}, d\right)$ have either dimension $d / 2$ if $d$ is even or dimensions $(d-1) / 2$ and $(d+1) / 2$ if $d$ is odd. This explains why the bound on $N(k)$ in Theorem 4.15 does not change if $k \geq d / 2$.

(ii) If $P$ is a simplicial $d$-polytope with $f_{0} \geq d+2$ vertices, then Theorem 4.15 gives for its number of empty edges

$$
N(1) \leq \begin{cases}f_{0}\left(f_{0}-3\right) / 2 & \text { if } d=2 \\
\left(\begin{array}{c}
f_{0}-d \\
2
\end{array}\right) & \text { if } d \geq 3\end{cases}
$$

If $d=2$, the bound is always attained because $f_{0}\left(f_{0}-3\right) / 2$ is the number of "missing diagonals" of a convex $f_{0}$-gon. The results in [24] (see also Remark 9.9 of [16]) provide that the bound is sharp for stacked $d$-polytopes for all $d \geq 2$.

Remark 4.19 Recall that the $k$-skeleton of an arbitrary $d$-polytope $P$ is the set of all faces of $P$ whose dimension is at most $k$. Perles [18] has shown:

The number of combinatorial types of $k$-skeleta of $d$-polytopes with $d+g_{1}+1$ vertices is bounded by a function in $k$ and $g_{1}$.

In [10] Kalai gave a new proof of this result that relies on the concept of missing faces. Indeed, in the simplicial case one concludes by using a bound on $N(k)$ because the $k$-skeleton of a simplicial polytope is determined by its set of empty simplices of dimension $\leq k$. 
In [10] Kalai sketches an argument showing that the number of empty simplices can be bounded with very little information on the $g$-vector. Below, we slightly correct Theorem 3.8 of [10] and give explicit bounds. We use Notation 3.1.

Theorem 4.20 Fix integers $j \geq k \geq 1$ and $b \geq 0$. Let $P$ be a simplicial d-polytope $P$ with $g_{k} \leq b$ where we define $g_{i}=0$ if $i>u$. If $d \geq j+k$, then the number of empty $j$-simplices of $P$ is bounded by

$$
\begin{cases}b^{\langle k, j-k+1\rangle} & \text { if } j<d / 2, \\ b^{\langle k, j-k+1\rangle}+b^{\langle k, j-k\rangle} & \text { if } j=d / 2, \\ b^{\langle k, d-j-k\rangle} & \text { if } j>d / 2\end{cases}
$$

Proof We have to bound $\beta_{1, j+1}^{K}(K[P])$. By Corollary 4.8, $P$ has no empty $j$-simplices if $u+1 \leq j \leq d-u-1$. Thus, we may assume that $1 \leq j \leq u$ or $d-u \leq j \leq d-1$.

Case 1 . Assume $1 \leq j \leq u \leq d / 2$. Then Corollary 4.6 provides if $j<d / 2$,

$$
\beta_{1, j+1}^{K}(K[P]) \leq g_{j}^{\langle j\rangle}-g_{j+1} .
$$

Using Lemma 4.13, we see that the bound is maximized if $g_{j+1}=0$ and $g_{j}$ is as large as possible. Since the $g$-vector is an O-sequence, we get $g_{j} \leq g_{k}^{\langle k, j-k\rangle} \leq b^{\langle k, j-k\rangle}$. Our claimed bound follows.

If $j=d / 2$, then we get $j=u=d / 2$. Hence Corollary 4.6 gives

$$
\beta_{1, j+1}^{K}(K[P]) \leq g_{j}^{\langle j\rangle}+g_{j}
$$

Now the bound is shown as above.

Case 2. Assume $d / 2 \leq d-u \leq j \leq d-1$. By the above considerations, we may also assume that $j \neq d / 2$. Thus, Corollary 4.6 provides

$$
\beta_{1, j+1}^{K}(K[P]) \leq g_{d-j}-\left(g_{d+1-j}\right)_{[d+1-j]} .
$$

Using our assumption $d-j \geq k$, we conclude as above.

Remark 4.21 (i) Theorem 3.8 of [10] the existence of bounds as in the above result is claimed without assuming $d \geq j+k$. However, this is impossible, as Case 2 in the above proof shows. Indeed, if $d-j<k$ and $d>j>d / 2$, then knowledge of $g_{k}$ does not give any information on $g_{d-j}$. In particular, $g_{d-j}$ can be arbitrarily large preventing the existence of a bound on $\beta_{1, j+1}^{K}(K[P])$ in terms of $g_{k}, j, k$ in this case.

For a somewhat specific example, fix $k=j=2$ and $d=3$. Then the Billera-Lee 3 -polytope with $g$-vector $\left(1, g_{1}\right)$ has $g_{1}$ empty 2 -simplices.

(ii) Note that the bounds in Theorem 4.20 are sharp if $g_{k}=b$. This follows from the proof.

If we only know that $d$ is large enough compared with $j$ and $k$, then we have the following weaker bound. 
Corollary 4.22 Fix integers $j \geq k \geq 1, b \geq 0$, and $d \geq j+k$. Then the number of empty $j$-simplices of every simplicial $d$-polytope with $g_{k} \leq b$ is at most $b^{\langle k, j-k+1\rangle}+$ $b^{\langle k, j-k\rangle}$.

Proof By Theorem 4.20, it remains to consider the case where $j>d / 2$. However, then $d-j<j$, thus $b^{\langle k, d-j-k\rangle} \leq b^{\langle k, j-k\rangle}$, and we conclude again by using Theorem 4.20.

Remark 4.23 Notice that the bound in Corollary 4.22 is independent of the number of vertices of the polytope and its dimension, provided the latter is large enough.

In essence, all the bounds on the number of empty simplices are bounds on certain first graded Betti numbers of the Stanley-Reisner ring of a simplicial polytope. As such, using Theorem 3.17, they can be extended to bounds for the first graded Betti numbers of any graded Gorenstein algebra with the Weak Lefschetz property. We leave this and analogous considerations for higher Betti numbers to the interested reader.

We conclude this note by pointing out some directions for future research:

Remark 4.24 (i) It is an open problem whether the upper bounds on the number of empty simplices of simplicial polytopes obtained in this paper extend to the case of empty pyramids of arbitrary polytopes. Recall that an empty pyramid of a polytope $P$ is a subcomplex of the face complex of $P$ that consists of all the proper faces of a pyramid over a face of $P$.

More generally, it would be very interesting to investigate whether prescribing the (toric) $g$-vector (see Sect. 3.14 of [21]) bounds the number of empty simplices (or possibly even empty pyramids) of non-simplicial polytopes.

(ii) It is natural to wonder also about good lower bounds on the number of empty simplices for polytopes with a given $f$-vector. This problem seems difficult. For simplicial $d$-polytopes with $f_{0}$ vertices, Krull's Principal Ideal Theorem implies that the total number of empty simplices is at least $f_{0}-d$. Equality is true if $f_{0}-d \leq 2$, but in most other cases this bound seems far from being optimal.

Acknowledgements The author thanks Gil Kalai, Carl Lee, and Juan Migliore for motivating discussions, encouragement, and helpful comments. He also thanks the referees whose suggestions helped to improve the presentation.

\section{References}

1. Bigatti, A.: Upper bounds for the Betti numbers of a given Hilbert function. Commun. Algebra 21, 2317-2334 (1993)

2. Billera, L.J., Lee, C.W.: A proof of the sufficiency of McMullen's conditions for $f$-vectors of simplicial convex polytopes. J. Comb. Theory Ser. A 31, 237-255 (1981)

3. Bruns, W., Herzog, J.: Cohen-Macaulay Rings, rev. edn. Cambridge Studies in Advanced Mathematics, vol. 39. Cambridge University Press, Cambridge (1998)

4. Gotzmann, G.: Eine Bedingung für die Flachheit und das Hilbertpolynom eines graduierten Ringes. Math. Z. 158, 61-70 (1978)

5. Harima, T.: Characterization of Hilbert functions of Gorenstein-Artin algebras with the Weak Stanley property. Proc. Am. Math. Soc. 123, 3631-3638 (1995) 
6. Harima, T., Migliore, J., Nagel, U., Watanabe, J.: The Weak and Strong Lefschetz properties for Artinian $K$-algebras. J. Algebra 262, 99-126 (2003)

7. Herzog, J., Hibi, T.: Componentwise linear ideals. Nagoya Math. J. 153, 141-153 (1999)

8. Hulett, H.: Maximum Betti numbers of homogeneous ideals with a given Hilbert function. Commun. Algebra 21, 2335-2350 (1993)

9. Kalai, G.: Rigidity and the lower bound theorem. Invent. Math. 88, 125-151 (1987)

10. Kalai, G.: Some aspects of the combinatorial theory of convex polytopes. In: Polytopes: Abstract, Convex and Computational, Scarborough, ON, 1993. NATO Adv. Sci. Inst. Ser. C Math. Phys. Sci., vol. 440, pp. 205-229. Kluwer, Dordrecht (1994)

11. Kalai, G.: Polytope skeletons and paths. In: Goodman, O'Rourke (eds.) Handbook of Discrete and Computational Geometry. CRC Ser. Discrete Math. Appl., pp. 331-353. CRC, Boca Raton (1997)

12. Macaulay, F.S.: Some properties of enumeration in the theory of modular systems. Proc. Lond. Math. Soc. 26, 531-555 (1927)

13. McMullen, P.: The maximum number of faces of a convex polytope. Mathematika 17, 179-184 (1970)

14. McMullen, P.: The number of faces of simplicial polytopes. Israel J. Math. 9, 559-570 (1971)

15. McMullen, P.: On simple polytopes. Invent. Math. 113, 419-444 (1993)

16. Migliore, J., Nagel, U.: Reduced arithmetically Gorenstein schemes and simplicial polytopes with maximal Betti numbers. Adv. Math. 180, 1-63 (2003)

17. Pardue, K.: Deformation classes of graded modules and maximal Betti numbers. Ill. J. Math. 40, 564-585 (1996)

18. Perles, M.: Truncation of atomic lattices, unpublished manuscript (around 1970)

19. Stanley, R.: Hilbert functions of graded algebras. Adv. Math. 28, 57-82 (1978)

20. Stanley, R.: The number of faces of a simplicial convex polytope. Adv. Math. 35, 236-238 (1980)

21. Stanley, R.: Enumerative Combinatorics, vol. 1. Cambridge Studies in Advanced Mathematics, vol. 49. Cambridge University Press, Cambridge (1997)

22. Terai, N., Hibi, T.: Computation of Betti numbers of monomial ideals associated with cyclic polytopes. Discrete Comput. Geom. 15, 287-295 (1996)

23. Terai, N., Hibi, T.: Some results on Betti numbers of Stanley-Reisner rings. Discrete Math. 157, 311-320 (1996)

24. Terai, N., Hibi, T.: Computation of Betti numbers of monomial ideals associated with stacked polytopes. Manuscripta Math. 92, 447-453 (1997) 